\title{
GESTÃO DE REDES DE INOVAÇÃO NOS ESCRITÓRIOS DE TRANSFERÊNCIA DE TECNOLOGIA NO CEARÁ: A IMPLEMENTAÇÃO DE UM MODELO DE GESTÃO COLABORATIVO
}

\author{
MANAGEMENT OF INNOVATION NETWORKS IN \\ TECHNOLOGY TRANSFER OFFICES IN CEARÁ: THE \\ IMPLEMENTATION OF A MODEL OF COLLABORATIVE \\ MANAGEMENT
}

\section{GESTIÓN DE REDES DE INNOVACIÓN EN LAS OFICINAS DE TRANSFERENCIA DE TECNOLOGÍA EN EL CEARÁ: LA IMPLEMENTACIÓN DE UN MODELO DE GESTIÓN COLABORATIVO}

Teresa Lenice Nogueira da Gama Mota

Pós-doutoranda no Programa de Pós-Graduação em Administração da Universidade Estadual do Ceará. Fortaleza - Ceará - BR. E-mail: <teresa_ mota@yahoo.com.br>.

Samuel Façanha Câmara Doutor em Economia pela Universidade Federal de Pernambuco. Professor Adjunto da Universidade Estadual do Ceará. Fortaleza - Ceará - BR. E-mail: <sfcamara2000@gmail. com $>$.

Gisele Aparecida Chaves Antenor

Mestranda no Programa de PósGraduação em Administração da Universidade Estadual do Ceará. Fortaleza - Ceará - BR. E-mail: <giseleantenor@gmail. com>.

Brenno Buarque de Lima Mestrando no Programa de PósGraduação em Administração da Universidade Estadual do Ceará. Fortaleza-Ceará-BR. E-mail: <brenno_buarque@ hotmail.com>.

\section{RESUMO}

Este artigo possui o objetivo de abordar como se deu a implementação de um modelo de gestão inteligente de rede na Redenit-CE, a partir de um estudo empírico nos escritórios de transferência de tecnologia (ETTs) das Instituições de Ciência e Tecnologia (ICTs) localizadas no estado do Ceará, Brasil. A originalidade da contribuição consiste na implementação de um modelo baseado na teoria da complexidade em uma rede de inovação colaborativa. $O$ trabalho foi realizado a partir da pesquisa-ação, na qual também foram conduzidas entrevistas com atores da rede. Assim, concomitante à implementação do modelo teórico, no qual um dos resultados foi o Plano de Ação da Rede, realizaram-se atividades de gestão, como diagnóstico e planejamento estratégico da rede, com o envolvimento de representantes de cada um dos ETTs. Dos resultados até o momento alcançados, pode-se concluir que a proposta de gestão fundamentada na colaboração mostra-se aderente aos modelos de redes não hierárquicas e capaz de maturar a gestão da Redenit-CE.

Palavras-chave: Gestão da Inovação. Transferência de Tecnologia. Redes. Colaboração.

\begin{abstract}
This paper aims to address the implementation of an intelligent network management model in Redenit-CE, based on an empirical study in the technology transfer offices (TTOs) of the Science and Technology Institutions (ICTs) located in the state of Ceará, Brazil. The originality of
\end{abstract}


the contribution consists of the implementation of a model based on a complex theory in a network of collaborative innovation. The work was carried out from the research-action, in which interviews were also conducted with actors of the network. Thus, concomitant with the implementation of the theoretical model, where one of the results was the Network Action Plan, management activities were carried out, such as diagnosis and strategic planning of the network, with the involvement of representatives of each of the TTOs. From the results achieved so far, it can be concluded that the management proposal based on collaboration has shown to be adherent to non-hierarchical network models and able to mature the management of Redenit-CE.

Keywords: Innovation Management. Technology Transfer. Networks. Collaboration.

\section{RESUMEN}

Este artículo tiene el objetivo de abordar como ocurrió la implementación de un modelo de gestión inteligente de red en la Redenit-CE, a través de un estudio en las oficinas de transferencia de tecnología (ETTs) de las Instituciones de Ciencia y Tecnología (ICTs), ubicadas en el estado de Ceará, Brasil. La originalidad de la contribución consiste en la implementación de un modelo basado en la teoría de la complexidad en una red de innovación colaborativa. El trabajo fue realizado a partir de la pesquisa-acción, además fueron conducidas entrevistas con personas de la red. Por lo tanto, concomitante a la implementación del modelo teórico, donde uno de los resultados fue el Plan de Acción de la Red, realizaron actividades de gestión, como diagnóstico y planeamiento estratégico de la red, con la participación de representantes de cada uno de los ETTs. De los resultados logrados hasta el momento, se puede concluir que la propuesta de gestión fundamentada en la colaboración tiene se mostrado adherente a los modelos de redes no jerarquizadas y capaz de madurar la gestión de la Redenit- CE.

Palabras-clave: Gestión de la Innovación. Transferencia de Tecnología. Redes. Colaboración.

\section{INTRODUÇÃO}

Os NITs, Escritórios de Transferência de Tecnologia (ETTs), denominados no Brasil de Núcleos de Inovação Tecnológica (NITs), tiveram seu conceito revisado no Novo Marco Legal brasileiro da Inovação, pela Lei 13.243 (BRASIL, 2016), e têm por finalidade a gestão da política institucional de inovação das Instituições de Ciência e Tecnologia (ICTs). Estes núcleos vêm se estruturando, visando cumprir suas missões com a otimização de recursos e a maior eficiência operacional possível, procurando, em muitos casos, atuar em rede na busca de melhores condições no compartilhamento de suas atividades. Esta atuação se constitui, assim, no formato de rede de inovação com múltiplos atores em uma organização associativa e sem vínculos hierárquicos entre seus agentes.

Este trabalho busca, através de um estudo empírico com a Redenit-CE, demonstrar como se deu o modelo de gestão inteligente de rede. As redes são ambientes de alta complexidade, nas quais os gestores possuem baixo nível de controle sobre os atores participantes. Isto também se aplica aos NITs, de modo que, como atores principais, necessitam interagir e colaborar com diversos outros atores, como suas ICTs, grupos de pesquisa, pesquisadores, gestores de spin-offs acadêmicas, e, em muitos casos, empresas e outras associações relacionadas.

A Redenit-CE é constituída pelos NITs das ICTs do estado do Ceará. No total, são vinte e dois núcleos de instituições públicas e privadas, de ensino e pesquisa, distribuídos em todo o estado. O intuito da Redenit-CE é promover a inovação através de estímulo à criação e a consolidação dos Núcleos de Inovação Tecnológica, contribuindo para o desenvolvimento do estado do Ceará. Ela atua em rede, conectando os NITs e promovendo a interação e a colaboração entre eles. Um dos desafios que se coloca para esta rede é a formatação de seu modelo de gestão, visto que a rede não pode atuar no modelo tradicional de gestão.

Neste sentido, o modelo/sistema de gestão da rede precisa trazer resultados relevantes, esperados e compartilhados por seus membros e pela sociedade, de forma que esta direção possa ser 
chamada de efetividade da governança da rede. Assim, o modelo de gestão precisa se adaptar às condições de rede complexa e, neste movimento, romper com os aspectos fundamentais dos modelos clássicos de gestão, baseados, fortemente, no controle, em busca de sua efetividade. Desta forma, considerando a Redenit-CE um ambiente complexo, o esquema conceitual do modelo foi elaborado a partir de embasamento nos princípios da Teoria da Complexidade.

Deste modo, o estudo da implementação desse modelo de gestão se faz relevante para a área de estudos das redes de inovação. Muito se discute na literatura da área sobre a importância de modelos de gestão que enfatizem os aspectos sociais, relacionais e colaborativos das redes (JONES; HESTERLY; BORGATTI, 1997; GULATI, 1995; PROVAN; FISH; SYDOW, 2007; CULLEN; JOHNSON; SAKANO, 2000; DYER; NOBEOKA, 2000; BALESTRIN; VERSCHOORE; PERUCIA, 2014). No entanto, não há evidências de estudos que relatem um caso empírico de implementação de um modelo de gestão colaborativo em determinada rede. Deste modo, este trabalho visa contribuir com essa lacuna da literatura.

A propositura de executar um modelo de gestão de redes precisa levar em conta que as redes evoluem no tempo e que estas são essencialmente dinâmicas. Uma das principais características da gestão inteligente de redes consiste na substituição da abordagem tradicional hierárquica pelo enfoque na gestão colaborativa, por meio da participação e do engajamento dos integrantes da rede, com a institucionalização das rotinas de cooperação (CAPALDO, 2007; BALESTRIN; VERSCHOORE, 2010; VALE JUNIOR; BORTOLASO; VERSCHOORE, 2010). Esta constatação, associada à complexidade envolvida nas relações existentes em redes distribuídas, demonstram que a gestão da Redenit-CE precisa considerar que seus atores aprendem com o passado e alteram seus comportamentos ao longo do tempo (MITLETON-KELLY, 2003; MCELROY, 2003; MISCHEN; JACKSON, 2008).

Considerando a necessidade da adaptação e a implementação de um sistema de gestão na Redenit-CE com base nestes aspectos apresentados, este artigo possui o objetivo de abordar como se deu a implementação de um modelo de gestão inteligente de rede na Redenit-CE, a partir de um estudo empírico nos ETTs das ICTs localizadas no estado do Ceará, Brasil. O estudo foi realizado a partir do diagnóstico da rede e do seu modelo de gestão, apropriado a ambientes complexos e de baixo controle, denominado de modelo de Gestão Inteligente de Redes (GIR), desenvolvido, inicialmente, para a gestão de aglomerados urbanos complexos e inovadores (CÂMARA; PINTO; CARVALHO, 2014).

Este artigo divide-se em cinco seções, contando com esta introdução. Na segunda seção, aborda-se o norte teórico deste trabalho, tratando da relação entre redes, colaboração e inovação e desafio da gestão em ambientes complexos. Segue-se a apresentação do método utilizado no trabalho, acompanhado da discussão dos resultados. Por fim, são descritas as conclusões da pesquisa, nas quais são abordadas as principais contribuições do trabalho e recomendações de pesquisas futuras.

\section{REVISÃO DA LITERATURA}

\subsection{REDES, COLABORAÇÃO E INOVAÇÃO}

As redes organizacionais são constituídas como um conjunto de agentes com objetivos individuais que convergem por meio da colaboração (BALESTRIN; VERSCHOORE, 2008). São diversos os objetivos pelos quais profissionais e organizações trabalham no formato das redes: ganhos de escala e poder de mercado; aprendizagem e inovação; redução de custos e riscos; relações sociais (VERSCHOORE; BALESTRIN, 2006).

Diversos estudos abordam como a colaboração pode ser benéfica para diferentes agentes alcançarem seus objetivos. Axelrod (1984) demonstra como a colaboração é importante para indivíduos, organizações e até para as relações diplomáticas entre países. No que diz respeito às organizações que têm intenção de cooperar para atingir interesses mútuos, a constituição de uma rede interoganizacional possibilita a transferência de conhecimentos e tecnologia (TSAI, 2001; BRESCHI; MALERBA, 2005; WANG et al., 2014). 
Desta forma, tratando de maneira específica da inovação, as redes se constituem de forma a estimular a geração de inovação entre seus agentes. Nesta linha, diversos autores apontam como as redes de cooperação se organizam para proporcionar compartilhamento de conhecimentos e informações na geração de inovação (BRESCHI; MALERBA, 2005; BALESTRIN; VERSCHOORE, 2010; FUNK, 2014).

Sabe-se que a inovação é, cada vez mais, fator de competividade. Para que a inovação aconteça, a gestão da inovação, seja no âmbito das empresas ou das Instituições de Ciência e Tecnologia (ICT), desempenha papel fundamental. Se na empresa deve haver um ambiente instituído para gerir o processo de inovação, este se faz ainda mais necessário nas ICTs, nas quais há uma diversidade de conhecimentos e uma complexidade de organismos. Esse é o papel desempenhado pelos Escritórios de Transferência de Tecnologia (ETT). Por outro lado, para que haja uma otimização do conhecimento gerado nas ICTs, é fundamental que elas trabalhem em rede.

As redes, como as de ETTs ou de NITs, se apresentam como alternativa para estratégias de cooperação entre organizações com o objetivo de inovação e transferência de tecnologia (POWELL; KOPUT; SMITH-DOERR, 1996; TSAI, 2001; BALESTRIN; VERSCHOORE, 2010). Balestrin e Verschoore (2010) demonstram, por exemplo, como a transferência de conhecimento através da colaboração em rede faz com que as empresas possam adotar novas práticas de trabalho e produzir novos produtos, gerando aprendizado e inovação. Adicionalmente, Tsai (2001) aponta como organizações no contexto das redes podem melhorar a transferência de conhecimento e, consequentemente, induzir mais inovações e alavancar as performances através da capacidade absortiva e dos relacionamentos interorganizacionais.

Neste contexto, Capaldo (2007) trata sobre os desafios de gerir redes interorganizacionais. Segundo o autor, é preciso desenvolver e alavancar um conjunto de capacidades relacionais para que as empresas possam desfrutar do potencial estratégico de gerar inovação e transferir conhecimento através de rede de alianças. Neste sentido, Dyer e Nobeoka (2000) apontam como a confiança é importante para facilitar a transferência de conhecimento tácito no contexto de redes organizacionais. Jones, Hesterly e Borgatti (1997), na mesma linha, apontam como os aspectos relacionais, como reputação e sanções coletivas, são importantes para os mecanismos de gestão das redes.

Deste modo, estes estudos apontam como os aspectos relacionais e colaborativos são importantes para as redes de cooperação, especificamente as que possuem objetivos de inovação e transferência de conhecimento. O modelo de gestão de uma rede deve trazer resultados relevantes, esperados e compartilhados por seus membros e pela sociedade, de forma que esta direção possa ser chamada de efetividade da governança da rede.

Verschoore, Balestrin e Perucia (2014) elencam seis critérios para a gestão das redes de cooperação: estratégia, coordenação, liderança, estrutura, processos e relacionamento. Segundo os autores, o critério Estratégia visa analisar a orientação estratégica do modo como a rede formula e direciona suas ações estratégicas, desdobrando-as em planos de ações e metas; o critério Coordenação avalia como e por quais instrumentos a rede tem conseguido manter a unidade e o envolvimento do grupo; já a Liderança analisa a solidez dos líderes da rede, o incentivo à pró-atividade e à troca de informações, além do comprometimento pessoal dos associados.

A Estrutura, entendida como um mecanismo de suporte para o desenvolvimento da rede, é analisada do ponto de vista de sede para o atendimento dos participantes e a formação e manutenção de uma equipe de suporte para proporcionar a perenidade da rede. Por fim, o critério Processos examina como a rede gerencia, analisa e melhora seus fluxos de trabalho, e o relacionamento concentra-se na interação com os participantes internos e com os stakeholders externos.

A propositura de um modelo de gestão de rede precisa considerar que seus atores aprendem com o passado e alteram seus comportamentos ao longo do tempo (MITLETON-KELLY, 2003; MCELROY, 2003; MISCHEN; JACKSON, 2008), o que é importante para a formatação do modelo de gestão/governança. Para Provan e Kenis 
(2008), existem dois modelos de governança de redes: o modelo chamado de Participant-Governed Network (PGN), em que a rede é governada pelos seus participantes de maneira formal ou informal; e o modelo Network Administrative Organization (NAO), na qual a rede é governada por uma entidade administrativa à parte de seus membros. Os autores, no entanto, não tratam sobre o fato de que existem redes que possuem diferentes características e formatos em seu modelo de gestão, de modo a mesclar e alternar o seu modelo entre o formato PGN e o formato NAO, demandando um maior nível de complexidade em sua gestão.

\subsection{GESTÃO DE REDES EM AMBIENTES COMPLEXOS}

Modelos clássicos de gestão são baseados, fortemente, no controle, em busca de sua efetividade. As redes precisam ter objetivos claros, mas, dinamicamente, variáveis e se definem não pela discricionariedade de lideranças emergentes, mas que se explicam pela apropriação das expectativas de seus atores. Este é um desafio que se coloca ao planejamento das redes, pois os instrumentos de gestão vinculados aos modelos tradicionais são baseados no controle e aplicados a sistemas fechados e hierárquicos, como as organizações empresariais.

A aplicabilidade de modelos de gestão tradicionais e inspirados nos paradigmas gerados para a aplicação nas empresas ou organizações, que se apresentam como sistemas mais fechados e hierárquicos, são diametralmente opostos às características complexas das redes. Carlisle e McMillan (2006), McKelvey (1997) e Levy (1994) enfatizam que a complexidade é uma abordagem que tem significativas implicações sobre as questões de gestão.

Nesta direção, Carlisle e McMillian (2006) propuseram uma classificação de ambientes complexos que descreve, entre outras características, os diferentes níveis de mecanismos de controle. Percebe-se que os sistemas se classificam desde: i) mecânicos; ii) hierárquicos; iii) de complexidade emergente; iv) totalmente randômicos e sem padrão. As redes se enquadram em ambientes de complexidade emergente em direção a sistemas randômicos e sem padrão, que apresentam mecanismos frágeis ou inexistentes de controle. Esta constatação associada à complexidade envolvida nas relações existentes em redes conduz à adoção do conceito de Sistemas Complexos Adaptativos, o qual dá base ao modelo de gestão adotado na pesquisa.

O framework do modelo de gestão de redes investigado pela pesquisa é baseado em um modelo proposto para um modelo de gestão inteligente de cidades, desenvolvido por um grupo de professores do Programa de Pós-Graduação da Universidade Estadual do Ceará (CÂMARA; PINTO; CARVALHO, 2014). Este modelo foi proposto para ser aplicado em aglomerados urbanos, ambientes de alta complexidade. As redes, como ambientes de dinâmica evolucionária e complexa, também se apresentam como sistemas de baixo nível de controle sobre os seus atores e componentes. Pode-se perceber pela Figura 1 que o modelo, claramente, possui uma relação domínio-imagem biunívoca entre as duas dimensões consideradas nele.

Figura 1 - Modelo de Gestão Inteligente de Redes (GIR)

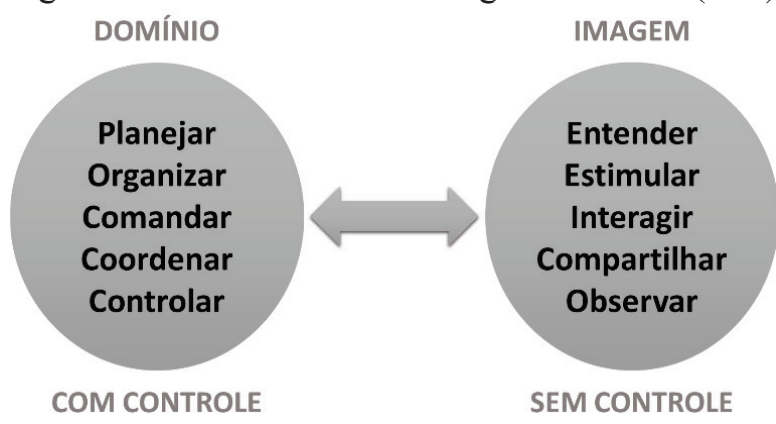

Fonte: (CÂMARA, PINTO; CARVALHO, 2014, p. 12).

O modelo GIR (Gestão Inteligente de Redes) propõe essas novas funções administrativas (Entender, Estimular, Interagir, Compartilhar e Observar), que não exigem, no limite, nenhum controle sobre o sistema a ser administrado. Embora pareça estranha esta afirmação, a essência dessa proposta é administrar sem controle. Este modelo tem como base a gestão em ambientes complexos.

Existem algumas pesquisas que abordam quais seriam os mecanismos de gestão adequados aos ambientes com estas especificidades, nos quais os mecanismos de controle não são tão efetivos (STACEY; GRIFFIN; SHAW, 2000; STRE- 
ATFIELD, 2001; STACEY; GRIFFIN, 2005). Esta forma de gerir se aplica ao ambiente das redes, que se caracteriza como de baixo controle. Assim, as funções propostas colocam, no centro da lógica de gestão destes sistemas (no caso desta pesquisa, as redes), a coevolução dos seus diferentes atores e não a tentativa de controle de um sobre o outro.

Desta forma, a função Entender é proposta como forma de acumular conhecimento sobre a evolução dos diferentes atores e as ligações entre os mesmos, sem aspectos reducionistas a priori, uma vez que a complexidade desses sistemas diz que variáveis aparentemente menos importantes podem influenciar, significativamente, na evolução de todo o sistema (CAPRA, 1996) e, diferentemente de sua função no conjunto domínio, a função planejar não deve ter a pretensão de conhecer e controlar o futuro, mas entender a evolução das diferentes trajetórias (STACEY; GRIFFIN; SHAW, 2000).

A função estimular é uma alternativa à função clássica organizar, uma vez que não se propõe a controlar as estruturas, mas estimular direções que se estabeleçam para os gestores e para os outros atores do sistema complexo em uma trajetória coevolutiva. Estimular, no modelo GIR, significa induzir, sem forçar ou controlar (AGOSTINHO, 2003; MERALI; ALLEN, 2011), as trajetórias que possam ser consideradas como benéficas para os atores envolvidos, adaptando as mesmas à dinâmica dos objetivos da rede apropriados pelos seus atores.

Interagir, ao invés de comandar, através do convencimento de que pessoas não são controláveis, mormente em um ambiente de auto-organização como os sistemas complexos (TÔRRES, 2009; STACEY; GRIFFIN, 2005; THIETART; FORGUES, 2011). A interação por meio da colaboração passa a ser a lógica predominante em situações evolutivas que emergem em trajetórias de longo prazo como estratégias dominantes de sobrevivência (AXELROD, 1984).

Compartilhar se coloca como uma função que, diferentemente da função coordenar, não se apresenta como ação de controlar as atividades a serem desenvolvidas e seus recursos. Ao contrário, compartilhar significa estabelecer uma lógica de uso de recursos não excludentes e compartilha- dos, principalmente as informações e o conhecimento (JONES; HESTERLY; BORGATTI, 1997; POWELL, 2003), promovendo as trajetórias coevolutivas entre os diferentes atores.

Observar os sistemas complexos é diferente de controlar. É preciso considerar que "há ordem no caos", e o que ela precisa é ser observada e compreendida. Por isso, esta função define a essência deste modelo, pois considera que nos sistemas complexos o controle é prescindível, e observar significa fechar o ciclo das funções, observando o comportamento do sistema, o que é fundamental para entendê-lo e estimulá-lo adequadamente, interagindo com os atores e compartilhando os recursos.

Assim, estas funções se colocam como uma nova forma de administrar, na qual não se pretende exercer controle, mas estimular as trajetórias evolutivas. Estas funções, contudo, não existem para os sistemas no vácuo. Elas são adotadas considerando alguns princípios de sua aplicação, como o uso de Tecnologias da Informação e Comunicação (TIC) para consecução das novas funções, elevando o potencial de colaboração e o consequente empoderamento dos atores e a cocriação de soluções em rede.

\section{MÉTODO}

Este trabalho foi feito a partir de uma pesquisa-ação, isto é, voltada para ação e a intervenção do pesquisador no processo da organização da rede com objetivo de proporcionar mudanças, melhorias e construções colaborativa do conhecimento a partir da reflexão sobre as práticas e as mudanças, tal como configura a pesquisa-ação de acordo com Franzolin, Minghini e Lourenço (2013).

Segundo Thiollent (2009), a pesquisa-ação objetiva assessorar os participantes a identificar os problemas e planejar possíveis soluções. Neste trabalho, os pesquisadores atuaram diretamente na gestão da rede, bem como na implementação do novo modelo de gestão. Para French (2009), a pesquisa-ação emancipatória exige o envolvimento de todos os participantes igualmente, descartando relações hierárquicas entre praticantes e pesquisadores. O objetivo do pesquisador é, como facilitador das discussões e do diálogo, tornar-se um membro colaborativo do grupo de praticantes, reduzindo a distância entre os 
problemas identificados e as teorias que permitem explicá-los e resolvê-los.

Dessa forma, a pesquisa-ação sobre a implementação do modelo colaborativo de gestão da Redenit-CE, seguindo as etapas do método da pesquisa-ação, iniciou-se pela fase de reflexão inicial exploratória, que consistiu de uma análise do contexto em que os NITs se relacionavam em rede, de modo a perceber o modelo de gestão que caberia a referida rede. Isso foi feito a partir de análise por meio de entrevistas realizadas com os gestores e os atores da rede. Por meio dessas entrevistas, foi possível fazer um diagnóstico da rede, de modo a estabelecer o atual estágio dela e, a partir disto, agir em sua gestão, a partir de um novo modelo de gestão, baseado nos princípios de um ambiente complexo.

As entrevistas foram guiadas a partir dos seis critérios de gestão de redes elencados por Verschoore, Balestrin e Perucia (2014). Para cada critério foi atribuída uma pontuação, sendo ressaltadas algumas ações positivas e alguns aspectos que podem ser melhorados na rede. As entrevistas foram conduzidas a partir de treze itens pertencentes aos seis critérios. A pontuação dos resultados seguiu escala definida e aplicada no modelo de avaliação concebido pelo Grupo de Estudo sobre Redes Interorganizacionais (GeRedes) da Universidade do Vale do Rio dos Sinos - UNISINOS, o qual também participou, em parceria com os pesquisadores, da aplicação das entrevistas e da análise de resultados. A Tabela 1 apresenta os níveis de avaliação elaborados pelo GeRedes. conteúdo, de acordo com os princípios elencados por Bardin (2011). Com base nos resultados destas entrevistas, sendo esta primeira etapa da pesquisa-ação classificada como reflexão inicial exploratória, foi possível a constituição de um diagnóstico para estabelecer qual o momento atual do desenvolvimento da rede.

A partir deste diagnóstico foi feito o processo de planejamento da rede, com uma pesquisa aprofundada que considerou instrumentos de gestão, instrumentos estratégicos, instrumentos contratuais, instrumentos de tomada de decisão, instrumentos de integração e rotinas de cooperação. Em meio a esse processo ocorreu, também, uma oficina de planejamento estratégico envolvendo os representantes de todos os NITs e os principais stakeholders do sistema local de inovação. A partir deste processo foram elaboradas diretrizes para a constituição de um Plano de Ação para a rede, que foi definido para ser executado a partir de um modelo de gestão de ambientes de baixo nível de controle. Assim, optou-se por adaptar o modelo proposto por Câmara, Pinto e Carvalho (2014) para a gestão de redes, no qual foi adaptado na Redenit-CE como Modelo GIR.

Assim, a etapa de ação se iniciou com a adaptação e implementação do modelo de Gestão Inteligente de Rede (GIR), aglutinado em um Plano de Ação, considerando as estratégias, os objetivos gerais, as metas e os projetos e ações da Redenit-CE. Este Plano de Ação se deu por meio de uma matriz, feita a partir do cruzamento do Mo-

Tabela 1 - Níveis de avaliação do GeRedes

\begin{tabular}{c|c|c|c|c}
\hline $\begin{array}{c}\text { Nível 1 } \\
\text { Iniciante }\end{array}$ & $\begin{array}{c}\text { Nível 2 } \\
\text { Básico }\end{array}$ & $\begin{array}{c}\text { Nível 3 } \\
\text { Intermediário }\end{array}$ & $\begin{array}{c}\text { Nível 4 } \\
\text { Adequado }\end{array}$ & $\begin{array}{c}\text { Nível 5 } \\
\text { Referência }\end{array}$ \\
\hline $\begin{array}{c}\text { Ainda faltam } \\
\text { requisitos mínimos }\end{array}$ & $\begin{array}{c}\text { Estágio de } \\
\text { construção e } \\
\text { mapeamento }\end{array}$ & $\begin{array}{c}\text { Estágio de } \\
\text { aprendizagem }\end{array}$ & $\begin{array}{c}\text { Consolidação e } \\
\text { melhoria }\end{array}$ & $\begin{array}{c}\text { Rede totalmente } \\
\text { incrementada e } \\
\text { sistematizada }\end{array}$ \\
\hline
\end{tabular}

Fonte: os próprios autores, de acordo com pesquisa realizada na Redenit-CE a partir do modelo do GeRedes.

As entrevistas foram feitas em quatro encontros presenciais de 90 minutos cada, com integrantes da Redenit-CE, escolhidos por especificidade de ICT: uma universidade, uma incubadora de empresas de base tecnológica, um instituto de pesquisa industrial e um instituto de pesquisa agrícola. A análise das entrevistas se deu por análise de delo de Gestão Inteligente de Redes (GIR) com os critérios de gestão de redes elencados por Verschoore, Balestrin e Perucia (2014). Ao final da elaboração deste artigo, a execução do Plano de Ação da Rede ainda estava em seu início, portanto diversas ações não serão contempladas aqui. 


\section{RESULTADOS}

Os resultados desta pesquisa apresentam como se deu a implementação de um modelo de gestão de rede colaborativo, sendo o objeto de estudo a Redenit-CE. Os pesquisadores faziam parte da rede e atuavam, direta e/ou indiretamente, na gestão. Assim, em um momento no qual a rede estava passando por uma reestruturação para a implementação de um novo modelo de gestão, de modo a solucionar o problema de gerenciamento, os pesquisadores decidiram realizar esta pesquisa-ação como modo de relatar os resultados deste processo e contribuir com a área de estudos de redes, inovação e modelos de gestão colaborativos.

\subsection{DIAGNÓSTICO}

Os primeiros resultados em ergiram da reflexão inicial exploratória, realizada por meio das entrevistas com os atores da rede, utilizadas como base para a etapa de planejamento ou pesquisa aprofundada, quando se deu a formulação Plano de Ação da Rede. Esta primeira seção expõe os resultados das entrevistas e do diagnóstico elaborado a partir destas, bem como dos objetivos elaborados pelos membros a partir dos resultados do diagnóstico. A Figura 2 e a Figura 3 apresentam os resultados obtidos pela rede em cada critério de sua gestão. As maiores pontuações foram nos critérios de "Liderança" e de "Coordenação".

Figura 2 - Análise da Gestão

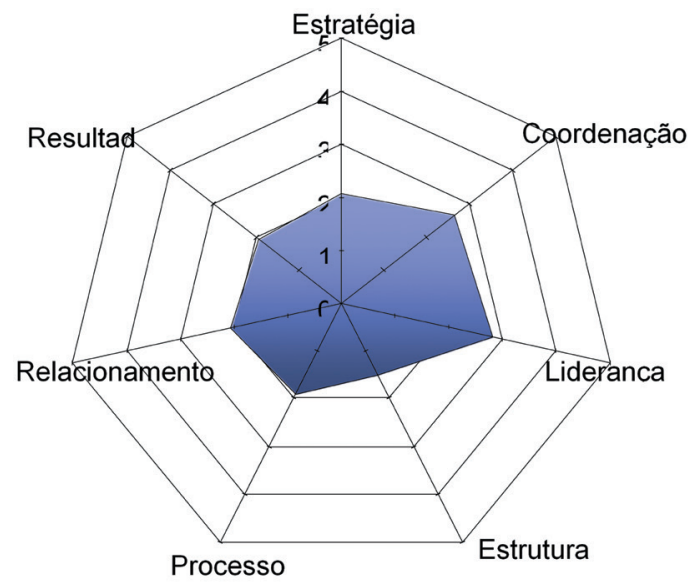

Fonte: os próprios autores.
A área destacada em azul representa o estágio de desenvolvimento da Redenit-CE, enquanto a área total da teia (nível cinco) representa o nível ótimo de desenvolvimento da gestão de uma rede, segundo os critérios avaliados. $\mathrm{Na}$ escala que vai de "1" a "5", a Redenit-CE obteve a pontuação de 2,12 pontos (média dos critérios), sendo classificada no Nível 2 de maturação, "Básico".

No critério Estratégia, a falta de um norte claro, que possa ser disseminado e incorporado pelos 18 NITs participantes, é um dos principais gargalos na gestão da rede. Não existe uma clara coesão de objetivos comuns entre os NITs que possa sustentar a Redenit-CE no longo prazo. Há, também, poucos indicadores que apontem os avanços que a rede tem ou não atingido em sua trajetória. $\mathrm{Na}$ Coordenação, a constância dos encontros mensais e a formalização de um regimento interno são considerados pontos positivos para a rede. No entanto, foi constatada a necessidade de elaboração de um código de ética para orientar a conduta dos representantes dos NITs dentro e fora da rede.

No quesito de Liderança, vale ressaltar que, desde a formação da rede, os líderes possuem uma postura aberta ao diálogo com os demais membros, além de sempre estimularem a pró-atividade e o compartilhamento de poder. Do lado negativo, foram observados, em alguns momentos, excesso de abertura ao diálogo, de modo a dificultar a tomada de decisão com eficiência. No critério Estrutura, destaca-se a definição de uma sede para o atendimento dos

Figura 3 - Pontuação da análise

\begin{tabular}{|l|l|}
\hline \multicolumn{2}{|l|}{ Resumo de Pontuação da Rede } \\
\hline Estratégia & 2,06 \\
\hline Coordenação & 2,63 \\
\hline Liderança & 2,80 \\
\hline Estrutura & 1,49 \\
\hline Processos & 1,89 \\
\hline Relacionamento & 2,06 \\
\hline Resultados & 1,92 \\
\hline Pontuação Final & 2,12 \\
\hline
\end{tabular}

Fonte: os próprios autores. 
participantes e a formação e a manutenção de uma equipe de suporte independente dos NITs. Foi percebido, também, que a Redenit-CE não necessita de um excesso de estrutura, pois deve centrar esforços nos aspectos que alavancam a cooperação entre os NITs.

Nos Processos, foi diagnosticado que falta um canal para o recebimento de sugestões de melhorias para o desenvolvimento da rede. Também se observou um hiato entre os NITs maduros e os NITs em consolidação, o qual chamou atenção para a necessidade de maior acompanhamento dos NITs em formação. No critério Relacionamento, percebeu-se bom relacionamento interno, com a preocupação da cooperação entre os membros. Também foi percebido bom relacionamento externo, com a relação às diferentes instâncias do governo estadual e a boa representatividade dos participantes em instâncias locais. No entanto, foi também percebido distanciamento entre as equipes técnicas que constituem os NITs.

Por fim, no quesito Resultados, os entrevistados evidenciaram os benefícios da rede em termos de representatividade, obtenção de recursos, aprendizagem e troca de informações. Ainda há espaço para avançar para a obtenção de benefícios para a rede, como em atividades e tecnologias que oneram os NITs e poderiam ser compartilhadas, proporcionando, assim, uma redução de custos. Outro fator citado pelos entrevistados foi a possibilidade de alinhar melhor as competências complementares que os NITs possuem, de modo a proporcionar mais benefícios pelo compartilhamento de competências.

\subsection{PROPOSTA DO MODELO GIR NA RE- DENIT-CE}

Considerando os referidos resultados das entrevistas, foi possível partir para a atuação na gestão da rede, trabalhando-se a partir de estratégias e de objetivos gerais, definindo-se metas, projetos e ações, conforme exemplificado na Tabela 2.
Assim, a equipe de pesquisadores, juntamente com outros membros da Redenit-CE, organizou e definiu diretrizes para a atuação na gestão da rede. No tocante aos objetivos comuns dos NITs para a rede, foram identificados e validados os seis itens a seguir: i) facilitar a institucionalização dos NITs em suas organizações; ii) ampliar a representatividade e o peso em negociações externas; iii) mapear talentos e integrar as competências complementares; iv) oferecer soluções coletivas às dificuldades comuns dos NITs; v) ampliar as pontes de conexão com o mercado e a sociedade; vi) aprimorar a gestão dos NITs em processos e pessoas.

Quanto aos objetivos gerais da Redenit-CE, um dos diagnósticos apontaram para: i) ampliar o impacto social do conhecimento gerado nas ICTs do Estado do Ceará por meio do fortalecimento e desenvolvimento dos NIT membros da Rede; ii) apoiar as iniciativas de NITs das ICTs do Estado do Ceará que visem a promoção da inovação, a criação de novos empreendimentos, a proteção da propriedade intelectual, a geração e a transferência de tecnologias; iii) consolidar-se como uma plataforma de intercâmbio e difusão de experiências, que impulsione boas práticas de gestão da inovação e do empreendedorismo dentro das ICTs do Estado do Ceará, incentivando a cooperação entre os membros da Redenit-CE, as empresas e os governos; iv) dar suporte às ações do Sistema Local de Inovação, contribuindo para aplicação efetiva da Lei de Inovação Federal e Estadual.

No tocante aos objetivos estratégicos para o período compreendido entre 2016 a 2020, foram obtidos os seguintes resultados: i) dar suporte à consolidação dos NITs em suas organizações; ii) ampliar a representatividade dos NITs e o peso em negociações externas; iii) mapear e difundir as competências científicas e tecnológi-

Tabela 2 - Plano de ação

\begin{tabular}{l|l|l|l} 
ESTRATEGIAS & OBJETIVOS GERAIS & METAS & PROJETOS (P) IAÇOES (A) \\
\hline $\begin{array}{l}\text { 1. Promover o } \\
\text { desenvolvimento a a } \\
\text { sustentabilidade da } \\
\text { Redeniţ-CE }\end{array}$ & $\begin{array}{l}\text { Estabelecer um modelo de } \\
\text { auto-geståo sustentável. }\end{array}$ & $\begin{array}{l}\text { Modelo de Geståo da Rede concebido até o } \\
\text { final de 2016. }\end{array}$ & $\begin{array}{l}\text { Conceber e implantar o Mlodelo de Gestāo } \\
\text { da Rede. (P) }\end{array}$ \\
& & & $\begin{array}{l}\text { Convênios e contratos em consonância } \\
\text { com o novo Marco Legal de CT\&I (A); }\end{array}$
\end{tabular}

Fonte: os próprios autores. 
cas das ICTs do Ceará; iv) oferecer soluções coletivas às dificuldades comuns dos NITs; v) facilitar e ampliar as conexões entre as ICTs, o setor produtivo e a sociedade; vi) aprimorar a gestão dos NITs por meio do fortalecimento dos seus agentes; vii) estimular e apoiar a criação de empreendimentos a partir das competências das ICTs membro.

No final deste planejamento da Redenit-CE, partiu-se para a fase inicial de implementação e avaliação do Modelo de Gestão Inteligente de Rede (GIR), no qual os objetivos traçados anteriormente foram aplicados no modelo GIR. O modelo é descrito e adaptado à Redenit-CE conforme a Tabela 3.

Tabela 3 - Estratégia utilizada no modelo GIR na Redenit-CE

\begin{tabular}{|c|c|c|c|c|c|}
\hline & Entender & Estimular & Interagir & Compartilhar & Observar \\
\hline $\begin{array}{l}\text { Princípios do } \\
\text { modelo }\end{array}$ & $\begin{array}{l}\text { Forma de } \\
\text { acumular } \\
\text { conhecimento } \\
\text { sobre a evolução } \\
\text { dos diferentes } \\
\text { atores e ligações } \\
\text { entre os mesmos }\end{array}$ & $\begin{array}{l}\text { Induzir trajetória } \\
\text { benéfica } \\
\text { aos atores } \\
\text { envolvidos, } \\
\text { adaptando-as } \\
\text { aos objetivos da } \\
\text { Rede }\end{array}$ & \begin{tabular}{|l} 
Interagir \\
por meio da \\
colaboração \\
em trajetórias \\
de longo prazo \\
como estratégias \\
de sobrevivência
\end{tabular} & $\begin{array}{l}\text { Estabelecer } \\
\text { lógica dos } \\
\text { recursos não } \\
\text { excludentes e } \\
\text { compartilhados, } \\
\text { principalmente } \\
\text { informações e } \\
\text { conhecimentos } \\
\text { entre os } \\
\text { diferentes atores }\end{array}$ & $\begin{array}{l}\text { Observar o } \\
\text { comportamento } \\
\text { do sistema, } \\
\text { entendo-o e } \\
\text { estimulando-o } \\
\text { adequadamente }\end{array}$ \\
\hline $\begin{array}{l}\text { Implementação } \\
\text { do modelo na } \\
\text { Redenit-CE }\end{array}$ & $\begin{array}{l}\text { Compreender o } \\
\text { diagnóstico, que } \\
\text { apontou falta } \\
\text { de estratégia, } \\
\text { de objetivos } \\
\text { comuns e } \\
\text { estruturação de } \\
\text { equipes }\end{array}$ & $\begin{array}{l}\text { Realizar } \\
\text { planejamento } \\
\text { estratégico, } \\
\text { com definição } \\
\text { de missão, } \\
\text { proposta de } \\
\text { valor, estratégias, } \\
\text { objetivos e metas }\end{array}$ & $\begin{array}{l}\text { Criar grupos para } \\
\text { implementação } \\
\text { do planejamento } \\
\text { estratégico } \\
\text { a partir da } \\
\text { identificação } \\
\text { de expertises } \\
\text { em reuniões } \\
\text { periódicas, } \\
\text { constituindo } \\
\text { células de } \\
\text { implementação }\end{array}$ & $\begin{array}{l}\text { Definir projetos } \\
\text { e ações capazes } \\
\text { de viabilizar o } \\
\text { planejamento } \\
\text { estratégico a } \\
\text { ser executado } \\
\text { pelos membros } \\
\text { da rede a partir } \\
\text { de células de } \\
\text { implementação }\end{array}$ & $\begin{array}{l}\text { Realizar reuniões } \\
\text { periódicas } \\
\text { para avaliação, } \\
\text { acompanhamento, } \\
\text { readequações e } \\
\text { estímulo dos } \\
\text { participantes }\end{array}$ \\
\hline
\end{tabular}

Fonte: os próprios autores.

Assim, após a adaptação do modelo GIR à Redenit-CE, passou-se para a fase de implementação, considerando o planejamento realizado, e dividindo as ações da rede de acordo com as dimensões de gestão de redes elencadas por Verschoore, Balestrin e Perucia (2014). Deste modo, o modelo de gestão da Redenit-CE se constituiu em uma matriz entre o modelo GIR e os critérios de dimensões de gestão de redes, expressada na Tabela 4. 
Tabela 4 - Plano de Ação da Redenit-CE, matriz do Modelo GIR e das Dimensões de Gestão de Redes

\begin{tabular}{|c|c|c|c|c|c|}
\hline \multirow{2}{*}{$\begin{array}{l}\text { Gestão de } \\
\text { Redes }\end{array}$} & \multicolumn{5}{|c|}{ Dimensões da Gestão Inteligente de Redes (GIR) } \\
\hline & Entender & Estimular & Interagir & Compartilhar & Observar \\
\hline Estratégia & $\begin{array}{l}\text { Conjunto de } \\
\text { indicadores de } \\
\text { desempenho } \\
\text { da rede }\end{array}$ & $\begin{array}{l}\text { Plataforma } \\
\text { Colaborativa; } \\
\text { Canais de } \\
\text { Comunicação: } \\
\text { estimulando } \\
\text { para trajetórias } \\
\text { específicas }\end{array}$ & $\begin{array}{l}\text { Plataforma } \\
\text { Colaborativa: } \\
\text { interação de } \\
\text { informações de } \\
\text { projetos de P\&D } \\
\text { e setor produtivo }\end{array}$ & $\begin{array}{l}\text { Plataforma } \\
\text { Colaborativa: } \\
\text { negociação de } \\
\text { colaboração } \\
\text { de recursos em } \\
\text { projetos de P\&D; } \\
\text { Vigilância } \\
\text { Tecnológica: } \\
\text { realizar prospecção } \\
\text { tecnológica, estudos } \\
\text { de mercado e busca } \\
\text { de anterioridade }\end{array}$ & $\begin{array}{l}\begin{array}{l}\text { Site da Rede: } \\
\text { conjunto de } \\
\text { indicadores; }\end{array} \\
\text { Canais de } \\
\text { comunicação: } \\
\text { mostrando ações } \\
\text { dos NITs; } \\
\\
\text { Portfólio de } \\
\text { Tecnologias }\end{array}$ \\
\hline Coordenação & $\begin{array}{l}\text { Articulação } \\
\text { com os NITs } \\
\text { para fornecer } \\
\text { conteúdo aos } \\
\text { indicadores; } \\
\text { Constância } \\
\text { de reuniões } \\
\text { ordinárias }\end{array}$ & $\begin{array}{l}\text { Articulação } \\
\text { para uso da } \\
\text { plataforma } \\
\text { colaborativa, } \\
\text { incentivando } \\
\text { projetos } \\
\text { colaborativos } \\
\text { entre os NITs; } \\
\text { Coordenação } \\
\text { itinerante } \\
\end{array}$ & $\begin{array}{l}\text { Articulação } \\
\text { para uso da } \\
\text { plataforma } \\
\text { colaborativa: } \\
\text { mostra dos } \\
\text { resultados da } \\
\text { plataforma } \\
\text { nas reuniões } \\
\text { ordinárias }\end{array}$ & $\begin{array}{l}\text { Articulação para } \\
\text { uso da plataforma } \\
\text { colaborativa: mostra } \\
\text { dos resultados da } \\
\text { plataforma nas } \\
\text { reuniões ordinárias, } \\
\text { enfatizando o } \\
\text { compartilhamento } \\
\text { de recursos }\end{array}$ & $\begin{array}{l}\text { Relatório de } \\
\text { desempenho da } \\
\text { rede anualmente } \\
\text { - realizada pela } \\
\text { coordenação }\end{array}$ \\
\hline Liderança & $\begin{array}{l}\text { Formação de } \\
\text { comissões } \\
\text { de trabalho } \\
\text { para ações e } \\
\text { projetos da } \\
\text { rede entre } \\
\text { os NITs com } \\
\text { coordenadores }\end{array}$ & $\begin{array}{l}\text { Formação de } \\
\text { comissões } \\
\text { nas reuniões } \\
\text { ordinárias, } \\
\text { identificando } \\
\text { lideranças } \\
\text { emergentes e } \\
\text { situacionais } \\
\end{array}$ & $\begin{array}{l}\text { Objetivos e } \\
\text { metas comuns a } \\
\text { serem cumpridas, } \\
\text { coletivamente, } \\
\text { pelos NITs das } \\
\text { comissões }\end{array}$ & \begin{tabular}{|l|} 
Líderes \\
(coordenadores) \\
das comissões, \\
articulando \\
compartilhamento \\
de recursos
\end{tabular} & $\begin{array}{l}\text { Divulgação dos } \\
\text { resultados das } \\
\text { comissões e } \\
\text { dos trabalhos } \\
\text { dos líderes } \\
\text { (coordenadores) }\end{array}$ \\
\hline Estrutura & $\begin{array}{l}\text { Conteúdos } \\
\text { de: Site da } \\
\text { rede; Fanpage } \\
\text { da rede; } \\
\text { Whatsapp } \\
\text { da rede; } \\
\text { Estrutura da } \\
\text { coordenação } \\
\text { itinerante; } \\
\text { NITDESK; } \\
\text { Regimento e } \\
\text { código de ética }\end{array}$ & $\begin{array}{l}\begin{array}{l}\text { Canais e } \\
\text { comunicações da } \\
\text { rede; }\end{array} \\
\text { Portfólio de } \\
\text { tecnologias; } \\
\text { Plataforma de } \\
\text { Colaboração }\end{array}$ & $\begin{array}{l}\text { Plataforma de } \\
\text { Colaboração }\end{array}$ & $\begin{array}{l}\text { Plataforma de } \\
\text { Colaboração }\end{array}$ & $\begin{array}{l}\text { Visualização } \\
\text { do Portfólio de } \\
\text { tecnologias; } \\
\text { Visualização da } \\
\text { Plataforma de } \\
\text { Colaboração }\end{array}$ \\
\hline
\end{tabular}




\begin{tabular}{|c|c|c|c|c|c|}
\hline Processos & $\begin{array}{l}\text { Alinhamento } \\
\text { estratégico } \\
\text { recorrente }\end{array}$ & $\begin{array}{l}\text { Processo do } \\
\text { NITDESK para } \\
\text { gerar indicadores } \\
\text { da rede; } \\
\text { Processo para } \\
\text { gerar indicadores } \\
\text { coletivos da rede; } \\
\text { Processo } \\
\text { de Mostra e } \\
\text { Negociação de } \\
\text { Tecnologia }\end{array}$ & $\begin{array}{l}\text { Processo de } \\
\text { lançamento das } \\
\text { informações } \\
\text { na Plataforma } \\
\text { Colaborativa }\end{array}$ & $\begin{array}{l}\text { Processo de } \\
\text { negociação de } \\
\text { compartilhamento } \\
\text { de recursos entre os } \\
\text { NITs; } \\
\text { Processo de } \\
\text { Vigilância } \\
\text { Tecnológica }\end{array}$ & $\begin{array}{l}\text { Processos de } \\
\text { montagem de } \\
\text { portfólio de } \\
\text { tecnologias e de } \\
\text { visualização da } \\
\text { Plataforma de } \\
\text { Colaboração }\end{array}$ \\
\hline Relacionamento & $\begin{array}{l}\text { Levantamento } \\
\text { e apresentação } \\
\text { dos projetos } \\
\text { colaborativos }\end{array}$ & $\begin{array}{l}\text { Reuniões } \\
\text { ordinárias; } \\
\text { Reuniões das } \\
\text { comissões; } \\
\text { Treinamentos e } \\
\text { capacitações; } \\
\text { Reuniões de } \\
\text { confraternizações }\end{array}$ & $\begin{array}{l}\begin{array}{l}\text { Reuniões } \\
\text { ordinárias; }\end{array} \\
\text { Reuniões das } \\
\text { comissões; } \\
\text { Treinamentos e } \\
\text { capacitações; } \\
\text { Reuniões de } \\
\text { confraternizações }\end{array}$ & $\begin{array}{l}\text { Reuniões ordinárias; } \\
\text { Reuniões das } \\
\text { comissões; } \\
\text { Treinamentos e } \\
\text { capacitações; } \\
\text { Reuniões de } \\
\text { confraternizações }\end{array}$ & $\begin{array}{l}\text { Visualizar se } \\
\text { os níveis de } \\
\text { relacionamento } \\
\text { da rede estão } \\
\text { em crescente- } \\
\text { análise da rede }\end{array}$ \\
\hline
\end{tabular}

Fonte: os próprios autores.

A Redenit-CE, a partir do diagnóstico realizado nas entrevistas e nas reuniões de planejamento dos membros, pode ser caracterizada como uma rede distribuída, com baixa centralidade e com ligações de baixa intensidade entre seus membros e de significante interesse social. Uma rede com essas características se encontra em uma zona cinza entre dois modelos identificados na literatura pertinente (PROVAN; KENIS, 2008; VENKATRAMAN; LEE, 2004): i) Participant-Governed Network (PGN), na qual a rede é governada pelos seus participantes, de maneira formal ou informal; e ii) Network Administrative Organization (NAO), governada por uma entidade administrativa à parte de seus membros.

Nesta perspectiva, a Redenit-CE possui o desafio de conviver com a complexidade inerente à governança distribuída em rede (PGN), bem como as dificuldades de uma entidade administrativa de se relacionar, de forma coordenada, com os membros da rede (NAO). Este desafio impõe ao modelo/sistema de gestão da rede características próprias, que permita à rede evoluir por um cami- nho que a faça cumprir com seu dinâmico papel social. A implementação de um novo modelo de gestão, baseado nos princípios da Teoria da Complexidade e nas dimensões da gestão de redes da literatura nacional, busca suprir as necessidades da Redenit-CE, de acordo com o seu ambiente peculiar e suas especificidades: um ambiente complexo, com baixo nível de controle e de grande potencial colaborativo e inovador.

\section{CONCLUSÕES}

O principal objetivo do presente trabalho consistiu em abordar como se deu a implementação de um modelo de gestão inteligente de rede colaborativo na Redenit-CE. Foi realizada uma pesquisa-ação, tendo início com entrevistas com membros da referida rede, ao que se seguiu ao planejamento e implementação de um novo modelo de gestão de rede colaborativo. Este novo modelo de gestão foi idealizado a partir da necessidade de adoção de um modelo que se adequasse à complexidade e às especificidades desta rede. Por fim, foi 
elaborado um Plano de Ação da Rede seguindo os princípios do modelo de gestão e adaptando-os a uma matriz com as principais dimensões de gestão de redes elencadas na literatura nacional.

Este trabalho buscou sanar uma lacuna da literatura no que diz respeito à proposição e a implementação de modelos de gestão de redes colaborativos, que podem ser aplicados em ambientes nos quais os gestores possuem baixo nível de controle sobre os atores e os recursos. Assim, nestes ambientes, pressupõe-se falha das dimensões tradicionais, baseadas no controle das organizações como base para seus processos de gestão. Nestes ambientes de alto grau de complexidade, a gerência, sob a perspectiva do controle, tende a proporcionar resultados pífios. Nestes ambientes, o modelo de gestão deve pautar-se sob princípios colaborativos, nos quais os membros possuem autonomia e as ações e os processos não dependem de uma hierarquia para ocorrer de forma fluida.

Este trabalho também aplica um método pouco usado na pesquisa em Administração, a pesquisa-ação, demonstrando como se deu a aplicação de um modelo de gestão sob a perspectiva dos gestores-pesquisadores. Este método se mostrou apropriado para abordar a implementação de um novo modelo de gestão em uma rede, na qual os pesquisadores participaram ativamente do processo.

Nos indícios coletados neste trabalho, conclui-se que a matriz elaborada a partir do modelo GIR e das dimensões de gestão de redes constituiu-se em um Plano de Ação capaz de maturar a gestão da Redenit-CE. No entanto, até a conclusão deste trabalho, as ações planejadas pelo modelo de gestão ainda não haviam sido implementadas por completo. Desta forma, uma das limitações deste trabalho é não poder avaliar as ações implementadas a partir do modelo de gestão formatado. No entanto, esta pesquisa cumpre seu objetivo, que é abordar como se deu a implementação de um modelo de gestão inteligente de rede colaborativo na Redenit-CE.

Recomenda-se, então, a realização de mais estudos na Redenit-CE, que possam relatar como a implementação do novo modelo de gestão causou impactos no longo prazo da rede. Também é recomendada a aplicação do modelo GIR em outras re- des de cooperação, bem como em outros ambientes com as características aqui supracitadas, de modo a verificar a viabilidade e a efetividade de sua aplicação em outros ambientes de baixo nível de controle. Por fim, também são recomendados estudos que abordem novos modelos de gestão de redes colaborativos, visto que o trabalho colaborativo e em rede é tendência nas organizações deste século.

\section{REFERÊNCIAS}

AGOSTINHO, Márcia E. Complexidade e organizações: em busca da gestão autônoma. São Paulo: Atlas, 2003.

AXELROD, R. The evolution of cooperation. London: Penguin Books, 1984.

BALESTRIN, Alsones; VERSCHOORE, J. R. Redes de Cooperação Empresarial: estratégias de gestão na nova economia. Porto Alegre: Bookman, 2008.

BALESTRIN, Alsones; VERSCHOORE, Jorge. Aprendizagem e inovação no contexto das redes de cooperação entre pequenas e médias empresas. Organizações \& Sociedade, Salvador, v. 17, n. 53, p. 311-330, abr./jun. 2010.

BALESTRIN, Alsones; VERSCHOORE, Jorge Renato; PERUCIA, Alexandre. A visão relacional da estratégia: evidências empíricas em redes de cooperação empresarial. BASE-Revista de Administração e Contabilidade da Unisinos, v. 11, n. 1, p. 47-58, 2014.

BARDIN, L. Análise de conteúdo. Tradução Luis Antero Reto, Augusto Pinheiro. São Paulo: Edições 70, 2011.

BRASIL. Lei $\mathrm{n}^{\mathbf{0}} \mathbf{1 3 . 2 4 3}$, de 11 de janeiro de 2016. Dispõe sobre estímulos ao desenvolvimento científico, à pesquisa, à capacitação científica e tecnológica e à inovação e altera a Lei no 10.973 , de 2 de dezembro de 2004, a Lei no 6.815 , de 19 de agosto de 1980 , a Lei no 8.666, de 21 de junho de 1993, a Lei no 12.462, de 4 de 
agosto de 2011, a Lei no 8.745, de 9 de dezembro de 1993, a Lei no 8.958, de 20 de dezembro de 1994, a Lei no 8.010, de 29 de março de 1990, a Lei no 8.032, de 12 de abril de 1990, e a Lei no 12.772, de 28 de dezembro de 2012, nos termos da Emenda Constitucional no 85, de 26 de fevereiro de 2015. Brasília, DF: Presidência da República, 2016. Disponível em: http://www.planalto.gov. br/ccivil_03/_ato2015-2018/2016/lei/113243.htm. Acesso em: 9 abr. 2017.

BRESCHI, Stefano; MALERBA, Franco. Clusters, networks and innovation. Oxford: Oxford University Press, 2005.

CÂMARA, Samuel; PINTO, Roberto; CARVALHO, Hermano 2014. Gestão Inteligente de Cidades: a complexidade e a inovação na gestão dos aglomerados urbanos. In: CONGRESSO DE ADMINISTRAÇÃO, SOCIEDADE E INOVAÇÃO, 4., 2014, Volta Redonda. Anais [...]. Rio de Janeiro: CASI, 2014.

CAPALDO, Antonio. Network structure and innovation: The leveraging of a dual network as a distinctive relational capability. Strategic management journal, v. 28, n. 6, p. 585-608, 2007.

CAPRA, Fritjof. The web of life: a new scientific understanding of living systems. [S.l.]: Anchor, 1996.

CARLISLE, Ysanne; MCMILLAN, Elizabeth. Innovation in organizations from a complex adaptive systems perspective. Emergence: Complexity \& Organization, v. 8, n. 1, p. 2-9, 2006.

CULLEN, John B.; JOHNSON, Jean L.; SAKANO, Tomoaki. Success through commitment and trust: the soft side of strategic alliance management. Journal of World Business, v. 35, n. 3, p. 223-240, 2000.

DYER, Jeffrey H.; NOBEOKA, Kentaro. Creating and managing a high-performance knowledge-sharing network: the Toyota case. Strategic management journal, v. 21, n. 3, p. 345-367, 2000.
FRANZOLIN, J. S.; MINGHINI, L.; LOURENÇO, M. L. Pesquisa-ação. Pesquisa qualitativa em administração: fundamentos, métodos e usos no Brasil. São Paulo: Atlas, 2013.

FRENCH, Steven. Action research for practising managers. Journal of Management Development, v. 28, n. 3, p. 187-204, 2009.

FUNK, Russell J. Making the most of where you are: geography, networks, and innovation in organizations. Academy of Management Journal, v. 57, n. 1, p. 193-222, 2014.

GULATI, Ranjay. Social structure and alliance formation patterns: a longitudinal analysis. Administrative science quarterly, v. 40, n. 4, p. 619-652, 1995.

JONES, Candace; HESTERLY, William S.; BORGATTI, Stephen P. A general theory of network governance: Exchange conditions and social mechanisms. Academy of management review, v. 22, n. 4, p. 911-945, 1997.

LEVY, David. Chaos theory and strategy: theory, application, and managerial implications. Strategic management journal, v. 15, n. S2, p. 167-178, 1994.

MCELROY, Mark W. The new knowledge management: complexity, learning, and sustainable innovation. [S.l.]: Routledge, 2003.

MCKELVEY, Bill. Perspective: quasi-natural organization science. Organization science, v. 8, n. 4, p. 351-380, 1997.

MERALI, Yasmin; ALLEN, Peter. Complexity and systems thinking. In: ALLEN, Peter; MAGUIRE, Steve; MCKELVEY, Bill (ed.). The SAGE handbook of complexity and management. $[S$. l.: s. n.], 2011. p. 31-52.

MISCHEN, Pamela A.; JACKSON, Stephen K. Connecting the dots: applying complexity theory, knowledge management and social network analysis to policy implementation. Public Administration Quarterly, v. 32, n. 3, p. 314-338, 2008. 
MITLETON-KELLY, Eve. Complex systems and evolutionary perspectives on organisations: the application of complexity theory to organisations. [S.l.]: Elsevier Science Ltd, 2003.

POWELL, Walter W.; KOPUT, Kenneth W.; SMITH-DOERR, Laurel. Interorganizational collaboration and the locus of innovation: networks of learning in biotechnology. Administrative science quarterly, v. 41, n. 1, p. 116-145, 1996.

POWELL, Walter. Neither market nor hierarchy. The sociology of organizations: classic, contemporary, and critical readings, v. 315, p. 104-117, 2003.

PROVAN, Keith G.; FISH, Amy; SYDOW, Joerg. Interorganizational networks at the network level: A review of the empirical literature on whole networks. Journal of management, v. 33, n. 3, p. 479-516, 2007.

PROVAN, Keith G.; KENIS, Patrick. Modes of network governance: structure, management, and effectiveness. Journal of public administration research and theory, v. 18, n. 2, p. 229-252, 2008.

STACEY, Ralph D.; GRIFFIN, Douglas; SHAW, Patricia. Complexity and management: fad or radical challenge to systems thinking?. [S.l.]: Psychology Press, 2000.

STACEY, Ralph D.; GRIFFIN, Douglas (ed.). A complexity perspective on researching organizations: taking experience seriously. [S.l.]: Taylor \& Francis, 2005.

STREATFIELD, Philip J. The paradox of control in organizations. [S.l.]: Psychology Press, 2001.

THIETART, Raymond-Alain; FORGUES, Bérnard. Complexity science and organization. In: ALLEN, Peter; MAGUIRE, Steve; MCKELVEY, Bill (ed.). The SAGE handbook of complexity and management. [S. l.: s. n.], 2011. p. 53-64.

THIOLLENT, Michel. Pesquisa-ação nas organizações. São Paulo: Atlas, 2009.
TÔRRES, José Júlio. Teoria da complexidade: uma nova visâo de mundo para a estratégia ${ }^{1}$. Revista Integra Educativa, v. 2, n. 2, p. 189-202, 2009.

TSAI, Wenpin. Knowledge transfer in intraorganizational networks: effects of network position and absorptive capacity on business unit innovation and performance. Academy of management journal, v. 44, n. 5, p. 996-1004, 2001.

VALE JUNIOR, Antunes. S. E. do; BORTOLASO, Ingrid Vargas; VERSCHOORE, Jorge Renato. Uma análise do relacionamento interno em redes de cooperação horizontais. In: ENCONTRO NACIONAL DE ENGENHARIA DE PRODUÇÃO - ENEGEP, 30., 2010, São Carlos, SP. Anais [...]. São Carlos: ENEGEP, 2010.

VENKATRAMAN, N.; LEE, Chi-Hyon. Preferential linkage and network evolution: A conceptual model and empirical test in the US video game sector. Academy of Management Journal, v. 47, n. 6, p. 876-892, 2004.

VERSCHOORE, Jorge Renato; BALESTRIN, Alsones; PERUCIA, Alexandre. Small-Firm Networks: hybrid arrangement or organizational form?. Organizações \& Sociedade, v. 21, n. 69, p. 275-291, 2014.

VERSCHOORE, Jorge R.; BALESTRIN, Alsones. Fatores competitivos das empresas em redes de cooperação. In: ENCONTRO DA ASSOCIAÇÃO NACIONAL DE PÓS-GRADUAÇÃO E PESQUISA EM ADMINISTRAÇÃO, 30., 2006, Salvador. Anais [...]. Salvador: Anpad, 2006.

WANG, Chunlei et al. Knowledge networks, collaboration networks, and exploratory innovation. Academy of Management Journal, v. 57, n. 2, p. 484-514, 2014. 